\title{
RESILIENCIA, SEXISMO Y VIOLENCIA DE PAREJAS EN ESTUDIANTES DE EDUCACIÓN SECUNDARIA DE LA REGIÓN DE MAULE, CHILE
}

\author{
Eugenio Saavedra G. \\ Docente e investigador, Universidad Católica del Maule, Chile \\ esaavedr@ucm.cl \\ José Cifuentes $\mathrm{N}$. \\ Ayudante de investigación, Escuela de Psicología \\ Universidad Católica del Maule, Chile \\ Jcifuentes334@gmail.com \\ Recibido 08/01/16 \\ Aprobado 25/03/16
}

\section{RESUMEN}

El presente trabajo da cuenta de las respuestas obtenidas en 226 estudiantes, hombres y mujeres, de la Séptima Región de Chile (Maule). Las variables a considerar fueron: resiliencia, sexismo y violencia. Para ello se administraron tres instrumentos a los sujetos durante el primer semestre del año 2015. Las escalas utilizadas fueron: Escala de Resiliencia para jóvenes y adultos SV-RES (Saavedra \& Villalta, 2008), Escala de Detección de Sexismo en Adolescentes DSA (Recio, Cuadrado \& Ramos, 2005) y la Escala de Tácticas de Conflicto Modificada MCTS (Neidig, 1986, en Muñoz y col. 2007). Se aplicó estadística descriptiva y se correlacionó las variables. Entre los principales resultados se obtuvo un nivel de resiliencia cercano al promedio, se describe un perfil marcado por el "exitismo" y el bajo desarrollo en el área afectiva. Por otra parte, se aprecia un nivel bajo de sexismo hostil y un sexismo benévolo por sobre el promedio. Referente a los estilos y estrategias de afrontamiento de conflictos, los jóvenes encuestados ocupan mayormente la "argumentación", sin embargo algunos de ellos resuelven sus conflictos a través de agresiones físicas moderadas y graves.

PALABRAS CLAVE: Resiliencia; Sexismo; Violencia; Jóvenes; Parejas; Maule; Chile 


\section{RESILIENCE, COUPLES SEXISM AND VIOLENCE IN SECONDARY SCHOOL STUDENTS OF MAULE, CHILE}

\section{SUMMARY}

This paper grasps the responses of 226 students, men and women, of the seventh region of Chile (Maule). The variables considered were: resilience, sexism and violence. Three instruments were administered to subjects in the first semester 2015. The scales used were: Resilience Scale for youth and adults SV-RES (Saavedra \& amp; Villalta, 2008), Sexism Scale Detection Teen DSA (Recio, Square \& Amp; Ramos, 2005) and Scale Modified Conflict Tactics MCTS (Neidig, 1986, Muñoz and cabbage. 2007). Descriptive statistics were applied and correlated variables as well. Among the main results, level close to the average resilience was obtained, a strong profile described by the "elitism" and under development in the affective area. Meanwhile, a low level was seen in hostile sexism and benevolent sexism above average. Regarding the styles and strategies coping with conflict, young respondents mostly occupy the "argument"; however some of them resolve their conflicts through moderate physical assaults.

Keywords: Resilience; Sexism; Violence; Youth; Couples; Maule; Chile.

\section{RESILIENCIA}

El concepto resiliencia aparece derivado de las experiencias en física e ingeniería, en donde se constatabala resistencia de ciertos materiales a las fuerzas ambientales y cómo los elementos afectados recuperaban su forma y atributos originales. Por otra parte, se observó al realizar estudios longitudinales en personas con alta vulnerabilidad, que un tercio de los niños presentaba una evolución favorable a pesar de sus circunstancias de pobreza y abandono, sin realizar ningún tipo de intervención adicional. (Werner et. al. 1989). Lo anterior llevó a pensar que existían características particulares en esos sujetos que los hacían más resistentes a los obstáculos y problemas, y les permitía seguir un desarrollo normal tanto físico como psíquico. De aquella época, pasando por las décadas del 80 y 90, apreciamos el surgimiento de diversos estudios y grandes esfuerzos por delimitar una definición de resiliencia (Saavedra, 2012). Dichas definiciones apuntaban básicamente a destacar tres elementos esenciales para considerar una conducta como resiliente: resistir una adversidad, sobreponerse y proyectarse a partir de ella.

Una definición pragmática de resiliencia sería entendida como la capacidad de una persona, o de un grupo, para desarrollarse bien, para seguir proyectándose en el futuro a pesar de acontecimientos desestabilizadores, de condiciones de vida difícil y de traumas a veces graves. (Manciaux, 2005). En esta dirección habría que señalar que la resiliencia nunca es 
absoluta. Es un proceso dinámico y nunca deberemos hablar de manera estática y determinista de "personas resilientes", sino más bien de sujetos que actúan de una manera resiliente (Saavedra, 2014).

Lo anterior se ve reforzado además por la constatación de que nuestras conductas, resilientes o no, varían según las circunstancias y contextos en que estamos (Saavedra, 2013). Influirán en que aparezca una conducta resiliente elementos temporales y espaciales, elementos históricos y sociales. En este sentido, la resiliencia es fruto de la interacción entre factores de riesgo y factores protectores presentes en el medio y en el sujeto mismo (Saavedra, 2011).

Boris Cyrulnik (1999) nos señala claramente que la resiliencia no es invulnerabilidad, que fluctúa a lo largo de la vida y por tanto no es una cualidad fija. En esa misma dirección, este autor nos destaca que una persona no puede actuar resilientemente estando en soledad, ya que la resiliencia se construye en la interacción con los otros, con el entorno y con su medio social (Cyrulnik, 1999). Este elemento de interacción e intersubjetividad, refuerza la idea de que la resiliencia no puede considerarse como un fenómeno puramente personal, sino que intervienen de manera sustancial quienes rodean e influyen en la persona (Saavedra, 2011).

En los años 80, se comienza estudiando preferentemente los elementos de riesgo y elementos protectores a la base de la resiliencia, pudiendo observar que un mismo elemento, persona o situación podían considerarse favorables o desfavorable para la persona. Dicho de otras forma, no existe un elemento que en si mismo sea de riesgo o protector, sino que dependerá del significado que tenga para cada sujeto (Rutter, 1987).
Los factores de riesgo pueden agruparse en 4: situación familiar, factores sociales/ ambientales, problemas crónicos de salud y amenazas vitales (Fortín y Bigras, 2000). Entre ellos cabe destacar contextos de pobreza, situaciones prolongadas de violencia, guerra, entre otros.

Por su parte los factores protectores pueden hallarse en los propios recursos internos, la familia y el ambiente social, laboral, educacional (Masten y Coastworth, 1998). Entre los elementos protectores observados con frecuencia en los sujetos, destaca el tener proyectos laborales o de estudio que impulsan a la persona a seguir hacia adelante en busca de alguna meta.

La idea de estudiar las potencialidades del ser humano ya habían sido difundida por las líneas humanistas de los años 50 . Al surgir con mayor fuerza esta concepción positiva de los sujetos contenida en el enfoque de la resiliencia, nos apartamos del estudio de las debilidades, las carencias y de los esfuerzos para compensar aquellos déficit. Ya no centraremos los esfuerzos en la distinción de la patología o en verificar las desviaciones de las personas respecto de una norma. Nos concentraremos en identificar las potencialidades $y$ posibilidades que un sujetos tiene frente a una situación de adversidad, construyendo una visión más positiva de la persona (Vanistendael, 1996). En este sentido, el concepto de riesgo, patología o desviación, conlleva a una estigmatización, resaltando lo negativo de la persona (Rassial, 1992).

Entre los elementos que facilitarían la generación de conductas resilientes podrían identificarse características al momentos de nacer, como la de presentar un "temperamento fácil y activo", como lo señala Werner y sus colaboradores (1992). También las variables de vínculo asociadas a los primeros años de vida de 
la persona serán fundamentales a la hora de generar conductas resilientes. Este vínculo podrá estar dado con la madre o quien reemplace esa función de cuidado y cercanía (Saavedra, 2011). En coherencia a lo anterior, ya desde la época de Rutter (1987) observábamos que personas sin problemas emocionales o de conducta presentaban más frecuentemente comportamientos resilientes.

Como una forma de ordenar algunos elementos que intervienen en la construcción de la conducta resilientes diremos que la persona requiere de algunos elementos de base, como relaciones de vínculo sólidas, sistemas de creencias, elementos identitarios y desarrollar roles activos en su vida (Saavedra, 2011).

En esta misma dirección, influirá en el desarrollo de una conducta resiliente la imagen que se tiene de sí mismo, la autoestima, el sentimiento de competencia frente a los problemas, el control percibido frente a la situación y la pertenencia a una red o colectivo (Saavedra, 2011). También será de suma importancia la visión que se tenga en torno al problema.
Una historia personal que desde la infancia muestre padres o adultos que confirman al niño y lo toman en cuenta, reforzará en éste un sentido de capacidad y la convicción de poder enfrentar situaciones de adversidad. Esa historia de enfrentamientos positivos reflejará en el niño la sensación de que el problema resulta abordable, al menos parcialmente o bien en su totalidad (Saavedra, 2011).

Por otra parte, hay un ecosistema familiar, político, económico, social y cultural que influye en nuestra capacidad para desarrollar conductas resilientes (Elbedour, 1993). La importancia de la redes al momento de enfrentar y superar las adversidades, generará un ambiente colectivo positivo que atenuará el dolor y desarrollará en el sujeto una visión del problema como más abordable, gracias a la contención y apoyo recibido (Saavedra, 2013).

Hoy en día, el equipo investigador en resiliencia de la Universidad Católica del Maule-Chile realiza estudios aplicando una matriz de resiliencia que abarca varios de los elementos mencionado anteriormente, a saber:

Tabla 1.- Matriz de resiliencia (Saavedra, 2014)

\begin{tabular}{|c|l|l|l|l|}
\hline & $\begin{array}{l}\text { Condiciones } \\
\text { de base }\end{array}$ & $\begin{array}{l}\text { Visión de sí } \\
\text { mismo }\end{array}$ & $\begin{array}{l}\text { Visión del } \\
\text { problema }\end{array}$ & $\begin{array}{l}\text { Respuesta } \\
\text { resiliente }\end{array}$ \\
\hline $\begin{array}{c}\text { Yo soy, yo } \\
\text { estoy }\end{array}$ & F1: Identidad & $\begin{array}{l}\text { F2: } \\
\text { Autonomía }\end{array}$ & $\begin{array}{l}\text { F3: } \\
\text { Satisfacción }\end{array}$ & $\begin{array}{l}\text { F4: } \\
\text { Pragmatismo }\end{array}$ \\
\hline Yo tengo... & $\begin{array}{l}\text { F5: } \\
\text { Vínculos }\end{array}$ & $\begin{array}{l}\text { F6: } \\
\text { Redes }\end{array}$ & $\begin{array}{l}\text { F7: } \\
\text { Modelos }\end{array}$ & $\begin{array}{l}\text { F8: } \\
\text { Metas }\end{array}$ \\
\hline Yo puedo & $\begin{array}{l}\text { F9: } \\
\text { Afectividad }\end{array}$ & $\begin{array}{l}\text { F10: } \\
\text { Autoeficacia }\end{array}$ & $\begin{array}{l}\text { F11: } \\
\text { Aprendizaje }\end{array}$ & $\begin{array}{l}\text { F12: } \\
\text { Generatividad }\end{array}$ \\
\hline
\end{tabular}


Como definiciones operacionales de estos elementos, podemos señalar:

Identidad: autodefinición básica, autoconcepto relativamente estable en el tiempo, caracterización personal.

Autonomía: sentimiento de competencia frente a los problemas, buena imagen de sí mismo, independencia al actuar. Control interno.

Satisfacción: percepción de logro, autovaloración, adaptación efectiva a las condiciones ambientales, percepción de desarrollo.

Pragmatismo: sentido práctico para evaluar y enfrentar los problemas, orientación hacia la acción.

Vínculos: condiciones estructurales que sirven de base para la formación de la personalidad. Relaciones vinculares, apego. Sistema de creencias.

Redes: condiciones sociales y familiares que constituyen un apoyo para el sujeto. Sistemas de apoyo y referencia cercanos y disponibles.

Modelos: personas y situaciones que sirven de guía al sujeto para enfrentar sus problemas. Experiencias anteriores que sirven de referente frente a la resolución de problemas.

Metas: objetivos definidos, acciones encaminadas hacia un fin. Proyección a futuro.

Afectividad: auto reconocimiento de la vida emocional del sujeto, valoración de lo emocional, características personales en torno a la vida emocional. Tono emocional, humor, empatía.

Autoeficacia: acción competente, efectividad, responsabilizarse por los actos, manejo de estrés frente a la tarea.
Aprendizaje: aprovechar la experiencia vivida, aprender de los errores, evaluar el propio actuar y corregir la acción.

Generatividad: capacidad de crear respuestas alternativas frente a los problemas, construir respuestas, planificar la acción (Saavedra, 2011).

\section{SEXISMO}

Uno de los principales resultados de las conductas violentas que los adolescentes repiten en sus vidas de pareja es encontrado en su infancia y cómo sus familias se relacionaban con dichas personas. Esta etapa de la vida está dedicada exclusivamente al desarrollo de la identidad propia y diferenciada. Existe un acuerdo en identificar como una de las causas más importantes de la violencia de género que siguen existiendo entre hombres y mujeres al status y al poder, y que el sexismo es utilizado para legitimar y mantener dichas diferencias (Díaz, 2003).

Una de las principales consecuencias de la cual se atribuyen estereotipos que asocian lo masculino con una serie de características que se denomina de forma genética agencia-instrumentalidad (que es la agresividad, competividad, acción, dureza, etc.) y lo femenino como expresividad-comunalidad (ternura, empatía, debilidad, dependencia, etc.) es más que nadapor lageneralización transcultural (Díaz, 2003). Y por esto la representación sexista puede actuar automáticamente al trasmitirse desde una edad temprana a los niños y niñas como deben comportarse y sus valores para identificarse.

Es preciso conocer algunas manifestaciones del sexismo, las cuales se dará a conocer en primera instancia en la adaptación escolar, rendimiento, auto-concepto y expectativas, las cuales parecen haber desaparecido, ya que por evaluaciones, como de auto 
informes, las adolescentes presentan un nivel de rendimiento y expectativas igual o superior al que manifiestan los adolescentes varones. En segunda instancia, el tiempo que dedican a sus diferentes actividades los adolescentes: ellosbuscaránmásactividades enfocadas al deporte y al estar en grupos en las que apenas hay comunicación verbal, en cambio las adolescentes presentarán más conductas pro sociales (Díaz, 2003). En tercera instancia, el género limita las estrategias emocionales produciendo diferencias evolutivas en la vulnerabilidad hacia los problemas emocionales. Así, en muchos temas podemos conocer algunas manifestaciones donde el sexismo está presente y donde estas conductas adoptadas se vas presentando desde la infancia y a través de las generaciones (Díaz, 2003).

Existe evidencia empírica (Formiga y Stangor, 2006 citado en Vaamonde, 2010) que plantea que los prejuicios se van construyendo a través de las interacciones sociales y que los valores humanos, por su potencial para orientar las decisiones, se perciben como una variable fundamental.

Para Vaamonde (2010), el concepto de sexismo se refiere a los prejuicios y comportamientos discriminatorios dirigidos a las personas en función a su género. Si bien a mediados del siglo XX los roles entre los hombre y mujeres han comenzado a ser más parecidos debido a la participación que han tenido en la sociedad, igualmente han quedado en el tiempo los abusos hacia ellas.

El sexismo es un constructo operacionalizado por dos dimensiones que se interrelacionan: uno es el sexismo hostil, que comprende las actitudes hostiles hacia las mujeres por su supuesta debilidad e inferioridad, y también está el sexismo benévolo, que se asocia con las actitudes positivas, lo que lleva a comportamientos pro sociales (Díaz, 2003). Glick y Fiske (en Díaz, 2003) sostienen que la ambivalencia sexista en hombres o mujeres se manifiestan con elevadas actitudes de ambos tipos, tanto de hostil como de benévolo. Algunas publicaciones han demostrado que los hombres presentan un nivel de sexismo hostil más elevado que las mujeres donde registran menos niveles de prejuicios (Díaz, 2003).

Factores de naturaleza sociocultural son una de las claves para el riesgo de la violencia de género, debido a su influencia de la trasmisión de modelos diferentes de masculinidad y feminidad entre los hombres y mujeres (Recio, Cuadrado y Ramos, 2007).

Los diferentes modelos se concretan en la construcción de identidades estereotipadas y sexistas basados en las creencias y actitudes de autoridad y dominio. En la actualidad se ponen de manifiesto diferentes formas tradicionales y nuevas de sexismo. Las nuevas formas pueden ser más difíciles de erradicar, no solo por su naturaleza sutil, sino también por un tono positivo que las hacen más aceptables (Recio, Cuadrado y Ramos, 2007).

Según Recio, Cuadrado y Ramos (2007), el sexismo hostil hace referencia al sexismo tradicional basado en una supuesta inferioridad de las mujeres como grupo, y el sexismo benévolo expresa un deseo por parte de los hombres de cuidar a las mujeres. Es un tipo de prejuicio hacia ellas basado en la visión estereotipada y limitada de la mujer, pero en un tono más afectivo. Estas conductas aumentan la dificultad de detectar o intervenir sobre él.

\section{VIOLENCIA EN PAREJAS JÓVENES}

Una de las tareas más complejas es llegar a una definición sobre violencia y lograr 
abarcar todo lo que implica este concepto en la práctica. Años de experiencia han llevado a entender que no existe una teoría que abarque todas las formas de violencia que existen, no se puede explicar todas de igual forma, todas ellas poseen diferentes miradas, tienen diferentes caras, tienen diferentes procesos (Sémelin, 1983, citado en Blair, 2009).

Variadas reflexiones $\mathrm{u}$ opiniones se remontan a los siglos XVIII y XIX, ya que en estos años es donde ocurre la mayor expansión y reflexión sobre la violencia debido a las guerras y las relaciones que existían entre países, etc. (Blair, 2009).

Blair (2009) hace mención a uno de los mejores análisis de la historia, a juicio propio, sobre la violencia que hace un llamado a que la "violencia es tan vieja como el mundo". Chesnais (1981 en Blair, 2009) hace referencia al uso extensivo y la falta de relativismo histórico, proponiendo una nueva conceptualización: habla sobre la escalada de la violencia donde deja en un campo libre cualquier tipo de interpretaciones parciales e inimaginables, y debido a esto la violencia ha llegado a designar todo choque, tensión $\mathrm{y}$ desigualdad. En sencillas palabras, para Chesnais, la noción de violencia es todavía imprecisa, elástica y sobre todo extensible a voluntad.

La violencia, en sentido estricto, es la de carácter físico. Es un ataque directo, corporal contra las personas y ella reviste un triple carácter: brutal, exterior y doloroso. Lo que se define es el uso del material de la fuerza, la rudeza voluntariamente cometida en perjuicio de alguien. Esta definición es una de la conclusiones que saca la autora y que da a conocer expresamente en su investigación (Blair, 2009).
Además, plantea que no es posible establecer un concepto de violencia unívoco y simple. Piensa que construyendo, decontruyendo y reconstruyendo los conceptos e interpretaciones con las cuales se trabaja en cada investigación, y proponiendo nuevas líneas de análisis, es posible trascender la descripción antropológica, política y/o sociológica de los fenómenos y establecer líneas generales capaces de dar un marco de inteligibilidad a esos fenómenos (Blair, 2009).

En la población adolescente se sitúa la franja de personas que está más expuesta a la violencia, y de modo especial es en el ambiente escolar donde se ve más afectado en sus relaciones, su calidad de clases y el desempeño académico (Abramovay, 2005). Los estudios señalan que los jóvenes de Latinoamérica que están en las edades de 15 a 24 años son los que están más expuestos a la violencia, ya sea como víctima o como victimarios (Abramovay, 2005).

Abramovay (2005) plantea, en concordancia con lo anteriormente señalado por otros autores, que el vocablo "violencia" posee múltiples significados, incorpora gran diversidad de sentidos definidos en términos históricos y culturales y que además incluyen muchas situaciones que van desde pequeñas infracciones y ataques a bienes materiales hasta las situaciones que pueden producir riesgos para la vida propia.

Pese a la complejidad del concepto, Abramovay (2005) plantea que existe un punto de consenso básico, y que dicho consenso consiste en que todo y cualquier acto de agresión física, moral o institucional dirigido contra la integridad de uno o de varios individuos es considerado como acto de violencia. 
La literatura especializada orientada de forma específica a la cuestión de la violencia escolar fue formulada a lo largo de los años, a partir de diferentes puntos de vista y de distintos focos de interés. Se plantea que la definición de violencia escolar es un fenómeno heterogéneo que rompe la estructura de las representaciones básicas del valor social: la de la infancia y la de la escuela, así como la de la propia sociedad. Aún más, las violencias practicadas en el universo escolar deben ser jerarquizadas, de forma que sean comprendidas y explicadas. Tal jerarquía se basa en la naturaleza de los actos en cuestión. Hay actos asociados a lo que es llamado violencia (robo, violencia sexual, daños físicos, crimen, etc.), y hay actos de violencia institucional y simbólica (violencia en las relaciones de poder) (Abramovay, 2005).

La jerarquía propuesta anteriormente permite que el fenómeno de la violencia escolar sea comprendido de la manera más ampliay diversificada posible. Es entendido como parte de las acciones que resultan de una ruptura del dialogo (intimidación, insultos, e infracciones leves). También la violencia es comprendida en plural como las violencias practicadas por aquello que Bourdieu (2001, en Abramovay, 2005) llama poder oculto o violencia simbólica.

En las últimas décadas, la violencia entre iguales en la adolescencia se producen en la escuela donde se desarrolla en torno a lo que se conoce como bulling, que suelen incluir burlas, amenazas, intimidación, agresión física, aislamiento sistemático e insultos, y tienden a originar problemas que se repiten y se prolongan en el tiempo, supone un abuso de poder apoyado generalmente de un grupo contra una víctima que se encuentra indefensa (Jalón, 2005).
Un grupo de adolescentes destaca que en el ocio, ellos declaran vivir menos situaciones de agresión entre iguales que en la escuela, con la excepción de las coacciones con amenazas o con armas. Las frecuentes situaciones de exclusión y humillación que hay en las escuelas parecen tener origen de la orientación a la violencia que ejercen a ambos contextos (Jalón, 2005).

Una de las principales características que presentan los agresores es que ellos se exhiben en situaciones sociales negativas -aunque todavía tienen amigos que les siguen en sus conductas violentas- con una acentuada tendencia a abusar de su fuerza. Suelen ser físicamente más fuertes que los demás, son impulsivos, con escasas habilidades sociales, baja tolerancia a la frustración, dificultad para cumplir normas y entre otras más (Jalón, 2005).

Una situación en común que también se presenta en los adolescentes agresores es que tienen menos disponibilidad de estrategias no violentas de resolución de conflictos. Están más de acuerdo con las creencias que llevan a justificar las conductas violentas y la intolerancia en distintos tipos de relajaciones. Una de sus principales dificultades es ponerse en el lugar de otra persona donde su razonamiento moral es el más primitivo. Están menos satisfechos que los demás con sus aprendizajes y con sus relaciones y son percibidos como personas arrogantes e intolerantes (Jalón, 2005).

Otro contexto donde se da y se ha descubierto violencia es en las parejas de este mismo rango de edad, que son entendidas como antecesores de la violencia en las relaciones posteriores más estables. Como señalan Muñoz, Andreu, Graña, O'Leary y González (2007), muchos datos avalan que la violencia suele darse 
en parejas jóvenes donde existe violencia psicológica como física, y que en muchos casos dentro de la pareja son vistos como comportamientos normales.

Uno de los instrumentos más utilizados para medir violencia en parejas adolescentes es la versión modificada de Conflicts Tactics Scale (M-CTS). Esta escala consta de 18 preguntas bidireccionales para el agresor como para la víctima con formato de respuesta likert.

Esta guía para la evaluación de la forma en que los individuos resuelven sus discrepancias con sus parejas posee 4 dimensiones, las cuales nos permiten identificar diferentes niveles de violencia en las parejas adolescentes: la dimensión 1, que se denomina Razonamiento/argumentación; la dimensión 2, que se llama Agresión verbal/psicológica; la dimensión 3, la cual es Agresión física leve; y por último, la dimensión 4 de Agresión física severa (Muñoz, Andreu, Graña, O’Leary y González, 2007).

Cada dimensión tiene su significado según su gravedad, de la menos grave a la más grave:

- Dimensión 1: Razonamiento/argumentación: es la forma menos agresiva de resolución de conflictos

- Dimensión 2: Agresión verbal/ psicológica: dirigida a la resolución de los conflictos a través de insultos, discusiones y amenazas verbales.

- Dimensión 3: Agresión física leve: reflejan las tácticas agresivas manifestadas a través del contacto físico o corporal con la víctima, pero que no implican daños o lesiones físicas graves.

- Dimensión 4: Agresión física grave: reflejan el uso de tácticas físicas cuya consecuencias son graves para la propia integridad de la parejavictimizada.

\section{METODOLOGÍA}

Objetivo: Describir y relacionar las variables Resiliencia, Sexismo y Violencia en parejas, en estudiantes de enseñanza secundaria de dos colegios de la región del Maule, Chile.

Muestra: Para desarrollar este estudio, se abordó una muestra intencional de 226 estudiantes, mujeres y hombres, de Enseñanza Media (Secundaria) de la ciudad de Talca, Chile. La muestra se logró obtener dadas las facilidades otorgadas en dos colegios de la región. Otros establecimientos se negaron a participar.

Instrumentos: Para la obtención de la información en torno a las variables estudiadas, se ocuparon tres instrumentos tipo cuestionarios:

1. Escala de Resiliencia para jóvenes y adultos SV-RES (Saavedra \& Villalta, 2008). Consta de 60 ítems que se valoran de 1 a 5 puntos.

2. Escala de Detección de Sexismo en Adolescentes DSA (Recio, Cuadrado \& Ramos, 2005). Consta de 26 ítems que se valoran de 1 a 6 puntos.

3. Escala de Tácticas de Conflicto Modificada MCTS (Neidig, 1986, en Muñoz y col., 2007). Consta de 36 ítems que se valoran de 1 a 5 puntos.

La administración de las escalas se llevó a cabo durante el primer semestre del año 2015.

Análisis: Los datos fueron sometidos a estadística descriptiva correlacional a través del programa estadístico SPSS, versión 18.0. Se graficó la información para su mejor comprensión. 


\section{RESULTADOS}

La muestra definitiva quedó conformada por 226 sujetos entre 14 y 19 años, con un promedio de edad de 16, 18 años. La distribución por sexo fue de 120 mujeres y 106 hombres.

Figura 1.- Distribución por sexo DISTRIBUCIÓN POR SEXO

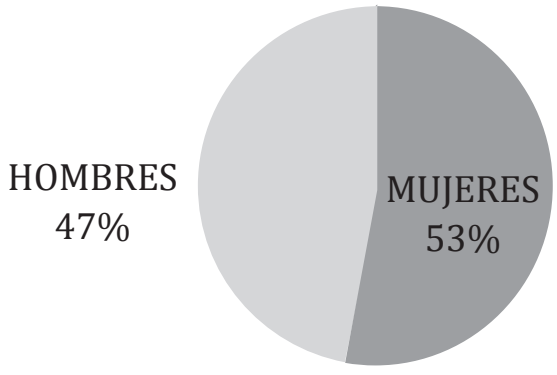

En cuanto a la variable resiliencia, se pudo observar que la muestra total se ubica en un percentil 50,5 con un puntaje promedio de 257,55 puntos.

Se determinó que las mujeres obtenían un puntaje de 255,29 puntos (percentil $47)$, en tanto los hombres lograron un promedio de 258, 44 puntos (percentil 51). Se estableció que no existen diferencias estadísticamente significativas.

Los puntajes por dimensión logrados en la escala SV-RES fueron los siguientes:

\section{Tabla 1.- Puntaje promedio por} dimensiones de resiliencia

\begin{tabular}{ll} 
Identidad & 21,24 \\
Autonomía & 21,23 \\
Satisfacción & 20,94 \\
Pragmatismo & 21,23 \\
Vínculos & 21,38 \\
Redes & 21,42 \\
Modelos & 22,70 \\
Metas & 21,28 \\
Afectividad & 20,48 \\
Auto eficacia & 21,85 \\
Aprendizaje & 21,71 \\
Generatividad & 21,93 \\
\hline
\end{tabular}

Podemos observar cómo las dimensiones Modelos, Generatividad y Autoeficacia destacan por sobre las otras, evidenciando lo que podríamos llamar un perfil centrado en el éxito, que se favorece del seguimiento de modelos y que son capaces de generar respuestas alternativas frente a las dificultades.

Del mismo modo, se evidencia que las dimensiones Afectividad y Satisfacción están disminuidas, reflejando con ello un menor desarrollo de la afectividad en estos jóvenes, como se observa en el siguiente gráfico.

\section{Figura 2.- Perfil de resiliencia por dimensiones}

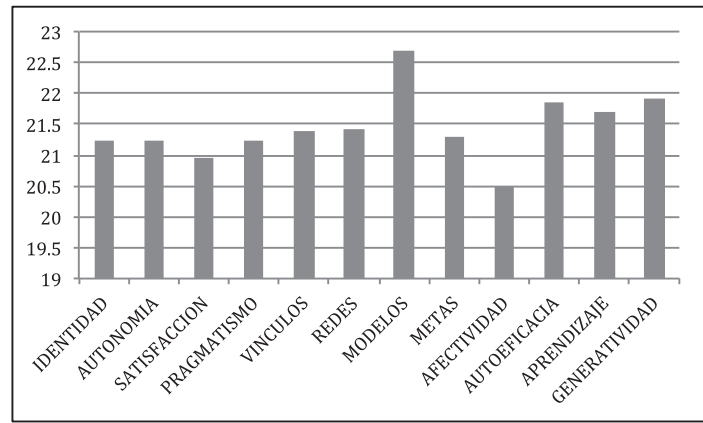

En torno al Sexismo, tomando en cuenta que los puntajes van de 1 a 6 , se obtiene para el "sexismo hostil" un promedio de 2,16 puntos, en tanto para el "sexismo benévolo" se obtiene un promedio de 3,26 puntos.

Según estos resultados, los encuestados muestran un bajo nivel de sexismo hostil, que se caracteriza por una carga afectiva negativa que supone una visión estereotipada y negativa de la mujer, donde se cometen abusos explícitos contra ellas.

Por su parte, el "sexismo benévolo" se encuentra en un nivel mayor, evidenciando que los encuestados tienen conductas respecto de las otras personas de mayor respeto y cordialidad. 
Al comparar el sexismo general por sexo, encontramos que los hombres puntúan 2,61 , en tanto las mujeres llegan a 2,83 , señalando un mayor promedio de sexismo en ellas.

En el caso de la argumentación, como primera dimensión para la situación de "victimas", se obtiene un puntaje de 2,8, de un total de 5. Esto los sitúa casi en la media del grupo, en donde predominaría una forma dialogada de resolver los conflictos. Por su parte la agresión psicológica en la situación de "victimas" alcanza a 2,27 puntos, refiriéndose a verbalizaciones descalificatorias, insultos, humillaciones, entre otras, como forma de enfrentar los problemas.

Para el caso de agresiones físicas moderadas, en donde se consideran "empujones" y otras agresiones físicas que no provocan heridas ni lesiones, el puntaje alcanza a 1,22 puntos en las "víctimas". En tanto las agresiones físicas graves en este mismo grupo alcanzan a 1,09 puntos, que si bien está en el límite inferior, llama la atención la existencia de estas agresiones en algunos sujetos.

Para la situación de los "victimarios", obtienen un puntaje de 2,82 en la estrategia de argumentación frente al conflicto, en tanto la agresión psicológica llega a 2,19 puntos. Por su parte, la agresión física moderada alcanza a 1,28 puntos y la agresión física grave a 1,11 puntos, que si bien estos dos últimos indicadores se sitúan en un puntaje bajo, de igual forma demuestra la existencia de dichas agresiones.

Al correlacionar las variables, a saber: resiliencia, sexismo benévolo, sexismo hostil, agresión física grave, agresión física moderada, argumentación y agresión psicológica, obtenemos correlaciones positivas altas en:
Tabla 2.- Correlaciones por variables

\begin{tabular}{lc}
\multicolumn{1}{c}{ Variables } & $\mathrm{r}$ \\
\hline $\begin{array}{l}\text { Sexismo hostil - sexismo benévolo } \\
\text { Víctima: agresión física grave - }\end{array}$ & 0,73 \\
$\begin{array}{l}\text { agresión física moderada } \\
\text { Victimario: agresión física grave - }\end{array}$ & 0,44 \\
agresión física moderada & \\
$\begin{array}{l}\text { Victimario: agresión física grave - } \\
\text { agresión física moderada }\end{array}$ & 0,52 \\
$\begin{array}{l}\text { Agresión física grave en victi- } \\
\text { mario - agresión física grave en }\end{array}$ & 0,80 \\
víctima \\
$\begin{array}{l}\text { Agresión física grave en victima- } \\
\text { rio - agresión física moderada en } \\
\text { víctima }\end{array}$ \\
\hline
\end{tabular}

La resiliencia no correlacionó con el resto de las variables, evidenciando que no existiría relación entre ésta y los distintos tipos de sexismo y agresiones. Del mismo modo, no correlacionó con la estrategia de argumentación en la resolución de conflictos.

\section{A MODO DE CONCLUSIÓN}

En términos de desarrollo de la respuesta resiliente, el grupo obtiene puntajes promedio, que lo sitúan en un percentil cercano a 50. En esta misma dirección, no encontramos diferencias estadísticamente significativas entre los promedios de resiliencia de hombres y mujeres.

Al analizar la resiliencia por dimensiones, observamos un perfil de tipo "exitista" en los adolescentes, caracterizado por respuesta de auto eficacia y generatividad, sumados al seguimiento de modelos.

Del mismo modo, se observa un perfil con un bajo desarrollo del área afectiva, lo que se ha observado en estudios anteriores con adolescentes.

Respecto del área de sexismo, se pudo constatar un bajo nivel de sexismo hostil que implica conductas abiertamente 
descalificatoria, insultos y actitudes de acoso. Por su parte, predomina el sexismo benévolo, caracterizado por conductas más bien pasivas de respeto y cordialidad.

Llama la atención en lo referido al sexismo general, que las mujeres obtienen un promedio mayor que los hombres encuestados.

La estrategia más ocupada para el enfrentamiento y resolución de conflictos es la argumentación, tanto para víctimas como victimarios.

Pudimos observar que a mayor intensidad de la agresión (psicológica, física moderada, física grave), disminuye el número de casos que ejercen este tipo de violencia. Sin embargo, si bien son pocos los casos que ejercen agresión física grave, es preocupante la existencia de ellos.

Respecto de las relaciones entre las variables, se concluye que éstas son las esperadas para el caso de sexismo y de violencia, sin embargo llama la atención la nula relación con la variable resiliencia.

En este sentido, resulta lógico y coherente con estudios anteriores una relación positiva entre los dos tipos de sexismos, a la vez que es esperable una correlación positiva entre la violencia psicológica y la física.

\section{FUENTES CONSULTADAS}

Abramovay, M. (2005). "Violencia en las escuelas: un gran desafío". Revista iberoamericana de educación (38), 53-66.

Blair, E. (2009). “Aproximación teórica al concepto de violencia: avatares de una definición". Revista Scielo (32), 9-33.

Cyrulnik, B. (1999). Un maravilleux malheur. Paris, Odile Jacob.
Díaz, M. (2003). "Adolescencia, sexismo y violencia de género". Revista Redalyc (84), 35-44.

Elbebour, S. (1993). "Ecological integrated model of children of war: individual and social Psychology". Child abuse and neglect (17), 805-819.

Fortin, L. \& Bigras, M. (2000). "La resilience des enfants, facteur de rusque, de protection et modeles theoriques". Practiques Psychologiques (1), 49-63.

Jalón, M. (2005). "La violencia entre iguales en la adolescencia y su prevención desde la escuela”. Psicothema, 17(4), 549-558.

Manciaux, m. (2005). La resiliencia: resistir y rehacerse. Gedisa, Barcelona.

Masten, A. \& Coastworth, J. (1998) "The developpmentof competence in favorable and unfavorable environments: lessons from research on successful children". American Psychologist 53(2), 205-220.

Muñoz, M., Andreu, J., Graña, J., O’Leary, D. y González, M. (2007). "Validación de la versión modificada de la Conflicts Tactics Scale (M-CTS) en población juvenil española". Psicothema 19(4), 693-698.

Rassial, J. (1992). Remarques sur le risque. Sante publique. 4, 23-25.

Recio, P., Cuadrado, I. y Ramos, E. (2007). "Propiedades psicométricas de la escala de detección de sexismo en adolescentes (DSA)". Psicothema 19(3), 522-528.

Rutter, M. (1987). "Psychosocial Resilience and Prospective Mechanisms". American Journal of Orthopsychiatry 57(3) 316-331.

Saavedra, E. (2011). La resiliencia desde una mirada post racionalista: dos historias de vida. Editorial Académica Española.

Saavedra, E. (2012). “Una aproximación al estado del arte dela investigación en resiliencia”. En Investigación orientada al cambio en Psicología Educacional (Jorge Catalán), Editorial Universidad de la Serena, Chile.

Saavedra, E. (2013). "Resiliencia familiar y mundo rural”. En Aproximaciones en Psicología Educacional (Cornejo, Morales, Saavedra y Salas). Universidad Católica del Maule, Chile.

Saavedra, E. (2014). “La construcción de la respuesta resiliente, un modelo y su evaluación”. En Nuevas miradas sobre la resiliencia (José María Madariaga) Editorial GEDISA, Barcelona. 
Vaamonde, J. (2010). "Valores y sexismo en adolescentes argentinos". Revista salud y sociedad, $1(2), 113-124$.

Vanistendael, S. (1996). La resilience ou le realism de l'esperance: blesse mais pas vaincu. BICE, Ginebra.
Vanistendael, S. (2002). La felicidad es posible. Gedisa, Barcelona.

Werner, E. \& Smith, R. (1992). Overcoming the odds: high risk children from birth to adulthood. Nueva York, Cornell University Press. 
